\title{
Abkürzungsverzeichnis und Organisationsregister
}

ADGB

AdsD

Agitprop

AJ

AP

Arbeiter-Turn- und Sportbewegung

Arbeiter- Turn- und Sportbund
Allgemeiner Deutscher Gewerkschaftsbund, Dachorganisation der Freien (Sozialdemokratischen) Gewerkschaften in der Weimarer Republik, vereinte in sich über 80 Prozent der gewerkschaftlich organisierten Arbeiter, 1922 ca. 7,9 Mill., 1929 4,9 Mill. Mitglieder.

Archiv der sozialen Demokratie (Friedrich Ebert-Stifung), Bonn, seit 1969 zentrales Institut der Sammlung von Dokumenten und Materialien zur deutschen und internationalen Sozialgeschichte unter besonderer Beriacksichtigung der Geschichte der Arbeiterbewegung.

Agitation und Propaganda; neben Politischen- und Organisationsleitern verfugten die Organisationseinheiten der KPD/KPO uber Agitprop-Leiter, die für die Werbearbeit der Partei zuständig waren.

Arbeiter-Jugend, Sammelbezeichnung für die seit 1904 auf lokaler Ebene entstehenden Aubeiterjugend-Vereine und die seit 1908 von der SPD zentral organisierten Jugendausschüsse um die gleichnamige Zeitschrift; trotz Abspaltung oppositioneller sozialistischer Jugendgruppen 1917/18 zu Beginn der Weimarer Republik größte linke Jugendorganisation, 192060000 Mitglieder, 192190000 , 1922 nach Rückkehr des rechten USPD-Flügels in die SPD Zusammenschluß der AJ und der „Sozialistischen Proletarierjugend“ der USPD zur SAJ.

Arbeiter-Partei, gegr. 1945 in Offenbach a.M. als Fortsetzung der vor 1933 lokal außerordentlich starken KPO/SAP, wurde aufgrund der Popularität ihres Vorsitzenden Heinrich Galm* und ihrer Aufbauleistung in Offenbach 1946 und 1948 mit 20 Prozent der Stimmen zur drittgrößten Partei; dagegen scheiterten $1947 / 48$ alle uberregionalen Ausdehnungsversuche, in Stuttgart und anderen Orten Württemberg-Badens blieb die AP Splitterpartei. Die Offenbacher $A P$, die sich seit 1947 zu einer marktwirtschaftlich orientierten Partei mit antibürokratisch-populistischen Tendenzen entwickelte, bestand bis zum Übertritt Galms zur SPD 1954 fort.

umfaßte die nach 1890 entstandenen proletarischen Turn- und Sportorganisationen, u. a. den Arbeiter-Turner-Bund, den ArbeiterRadfahrer-Bund „Solidarität“, den Arbeiter-Athletenbund und den Touristenverein „Die Naturfreunde“. Die ATuS-Bewegung, die dem bürgerlichen Hochleistungssport den solidarischen Mannschafts- und Massensport entgegensetzte, war für alle Arbeiter offen; de facto bildete sie in der Weimarer Republik neben Partei und Gewerkschaft die dritte Säule der sozialdemokratischen Aubeiterbewegung; 1928/29 erfolgte die Gründung eigenständiger kommunistischer Sportorganisationen.

mit 1928770000 Mitgliedern die bei weitem größte Arbeitersportorganisation der Weimarer Republik. 
Arbeiterwohlfahrt (AWO)

ASTA

A- und S-Rat

BBC

BDV

BMW

BP

Bremer Linksradikale

Bund der religiösen

Sozialisten

Deutschlands sozialdemokratische Wohlfahrtsorganisation, I919 von Marie Juchacz $^{*}$ gegr. mit dem Ziel, die kommunale und staatliche Wohlfahrtspflege im Sinne der Arbeiterschaft zu beeinflussen; wurde auf allen Gebieten der Sozialpolitik und der Sozialfursorge tätig; vor allem Frauen engagierten sich in der AWO bei der Betreuung von Kindern, Alten, Armen u. a.; 192424000 , 1930 I1 4000 , 1933 I 50000 ehrenamtliche Helferinnen und Helfer; nach 1945 wiedergegr. als parteiunabhängiger Wohlfahrsverband mit 1966 ; 100 hauptberuflichen Mitarbeitern und 78000 ehrenamtlichen Helfern.

Allgemeiner Studenten-Ausschuß, Exekutivorgan der Studentenschaft einer Hochschule oder Universität.

Arbeiter- und Soldaten-Rat, A- und S-Räte bildeten sich in der Revolution 1918/19 als Organe der revolutionären Arbeiter und Soldaten. Mehrheitlich aus SPD-Mitgliedern zusammengesetzt, übertrug die Mitte Dezember 1918 in Berlin tagende Reichskonferenz der Aund S-Räte die vollziehende und gesetzgebende Gewalt an den Rat der Volksbeauftragten unter Friedrich Ebert (SPD), auf derselben Konferenz stimmte eine große Mehrheit der Delegierten für eine möglichst schnelle Einberufung einer verfassunggebenden Nationalversammlung. Die A- u. S-Räte bestanden z.T. I9I9 als Kontrollorgane neben den alten Verwaltungsapparaten fort, politisch waren sie aber seit Frühjahr 1919 ohne Einfluß.

British Broadcasting Company, englische Rundfunkstation, sendete im Zweiten Weltkrieg deutschsprachige Programme, an denen sich auch deutsche Emigranten beteiligten.

Bremer Demokratische Volkspartei; gegr. im Dezember 1945, intendierte die Sammlung aller bürgerlichen Gruppen auf der Grundlage eines hanseatischen Liberalismus, bei der Bürgerschaftswahl im Okt. 194618 Prozent der Stimmen, 1948 Anschluß an die FDP.

Bayerische Motoren Werke, München

Bayernpartei, 1946 gegr., konservative föderalistische Partei mit überwiegend bäuerlich-mittelständischer Anhängerschaft in Oberund Niederbayern.

seit 1914 sich herausbildende sozialdemokratische Oppositionsgruppe um Johann Knief und Paul Frölich*, in ihrer Politik weitgehend von Lenin, Bucharin und insbesondere von Karl Radek beeinflußt; trat wie diese im Unterschied zur "Gruppe Internationale (Spartacus) " schon 1916 für die Spaltung der SPD und die Organisierung einer neuen Partei und Internationalen ein; ihr Einfluß beschränkte sich auf Bremen und Hamburg sowie auf kleinere Gruppen in Norddeutschland, im März 1917 Proklamation einer eigenständigen "linksradikalen Partei“, im November 1918 Umbenennung in "Internationale Kommunisten Deutschlands ${ }^{*}$, Ende 1918 zusammen mit der „Spartacus-Gruppe“ Gründung der KPD.

1926 gegr. Vereinigung mit dem Ziel, in Kirche und Arbeiterbewegung für einen religiös begründeten Sozialismus zu werben; 1930 1 0 25000 Mitglieder, meist Sozialdemokraten, darunter 200 Pfarrer; am stärksten verankert in einigen Industriedörfern, Klein- und Mittelstädten in Baden, Württemberg und Thüringen. 
Bund Technischer Angestellter und Beamter

CARE

\section{CDU}

CIA

CSR

CSSR

CSU

DAG

DDR

Deutsche

Friedensgesellschaft

Deutsch-jüdischer

Wanderbund

Kameraden

DFU

DGB

DKP gegr. 1919, dem freigewerkschaftlichen Allgemeinen Freien Angestelltenbund angeschlossen; umfaßte alle technischen Angestellten ohne Rücksicht auf ihre Vorbildung; 192760000 Mitglieder.

Abk. für: Cooperative for American Remittances to Europe, 1946 in den USA gegr., v. a. von privater Seite getragene Hilfsorganisation, die nach Ende des Zweiten Weltkrieges durch ihre Lebensmittelund Kleiderspenden wesentlich dazu beitrug, die Not in Europa zu lindern; in Deutschland bis 1960 tätig.

\section{Christlich-Demokratische Union Deutschlands}

Central Intelligence Agency, 1947 gegr. Zentralamt des amerikanischen Geheimdienstes

\section{Ceskoslovenská Republika, Tschechoslowakische Republik}

Ceskoslovenská Socialistická Republika, Tschechoslowakische Sozialistische Republik

\section{Christich-Soziale Union in Bayern}

Deutsche Angestellten-Gewerkschaft, gegr. im April 1949; versteht sich im Gegensatz zu den DGB-Gewerkschaften, die Arbeiter und Angestellte ohne Unterschied im Hinblick auf ihren beruflichen Status organisieren, als Vertreterin spezifischer Interessen der Angestellten.

\section{Deutsche Demokratische Republik}

1892 unter dem Einfluß Bertha von Suttners gegr. uberparteiliche pazifistische Vereinigung, 1914-1929 unter Leitung des Historikers und Friedensnobelpreisträgers (1927) Ludwig Quidde, danach Fritz Küsters; 1926 rd. 30000 Mitglieder, vertrat in ihrem Organ „Das andere Deutschland ${ }^{*}$ eine scharfe Kritik an der Tolerierungspolitik der SPD und der „pseudorevolutionären Katastrophenpolitik ${ }^{*}$ der KPD.

1919 gegr. auf nichtzionistisch-sozialistischer Grundlage

Deutsche Friedens-Union, 1960 gegr. Linkspartei, die für Abrüstung und eine Verständigung mit der DDR und den Staaten Osteuropas eintritt.

Deutscher Gewerkschaftsbund, Dachorganisation der 16 westdeutschen Industriegewerkschaften; im Unterschied zu den auf dem Berufsverbands-Prinzip beruhenden und an Parteien gebundenen Richtungsgewerkschaften der Weimarer Republik (sozialdemokratische, christliche, Hirsch-Dunkersche) wurde der DGB im Oktober 1949 als Zusammenschluß der in den westlichen Besatzungszonen nach 1945 ins Leben gerufenen Einheitsgewerkschaften gegründet; unabhängig von konfessionellen und parteipolitischen Bindungen umfaßt er Arbeiter und Angestellte nach ihrer Zugehörigkeit zu bestimmten Industriezweigen; 195 I 5,9 Mill. Mitglieder.

Deutsche Kommunistische Partei, 1968 von ehemaligen Funktionären der 1956 in der Bundesrepublik verbotenen $K P D$ gegründete $K P$ der Bundesrepublik. 
Deutsche Liga für Menschenrechte, im Ersten Weltkrieg unter der Bezeichnung „Bund neues Vaterland“ gegründet mit dem Ziel, einen schnellen Verständigungsfrieden herbeizuführen, $1922 \mathrm{Na}$ mensänderung und Beitritt zur Internationalen Liga für Menschenrechte; trat für eine demokratische soziale Republik in einem freien Europa ein. Wahrend der DLM in der Anfangsphase der Weimarer Republik Mitglieder der SPD, der DDP, der USPD und Parteilose angehorten, setzten sich die rd. 1000 Mitglieder 1931 vorwiegend aus parteilosen ,heimatlosen Linken zusammen, dementsprechend stand die DLM 1931/32 der SAP nahe.

DMV Deutscher Metallarbeiter-Verband, gegr. 1891, umfaßte nahezu alle Berufsgruppen der Metallbranche und stellte die bei weitem größte Einzelgewerkschaft des ADGB dar: 1922 1,6 Mill. Mitglieder, Ende 1924711000,1928884000 ; Ende 1932690000.

DNVP

Deutschnationale Volkspartei, 1918 gegr. nationalkonservative Partei, seit 1930 mit der NSDAP in der "Harzburger Front " verbunden, ermöglichte Hitler im Januar 1933 die Machtübernahme.

DP

Deutsche Partei, 1947 gegr., national-konservative Partei mit bäuerlich-mittelständischer Basis, im wesentlichen auf Norddeutschland beschränkt.

DReP Deutsche Rechtspartei, gegr. 1946, mit Hochburgen in Niedersachsen und Schleswig-Holstein, umfaßte einen national-konservativen und einen rechtsextremen Flügel um Adolf von Thadden, der 1949 die neofaschistische "Sozialistische Reichspartei“ gründete.

DVP Deutsche Volks-Partei, unter diesem Namen trat die 1946 gegr. FDP bis 1952 in Württemberg-Baden auf.

\section{EKKI}

FDGB

Exekutivkomitee der Kommunistischen Internationale

Freier Deutscher Gewerkschaftsbund, 1945 gegr. Einheitsorganisation der Industriegewerkschaften der SBZ/DDR.

FDJ

Freie Deutsche Jugend, im Rahmen der Volksfrontbestrebungen 1935/36 im Exil entstandenes Kartell der Jugendorganisationen der Arbeiterparteien; in ihm waren der $\mathrm{KJV}$, der SJV, die SAJ, die ISKJugend und später auch die Jugendorganisationen der österreichischen Sozialisten und Kommunisten zusammengeschlossen; bestand bis Kriegsausbruch. Nach 1945 Name der Jugendorganisation der SED.

FDP

Freidenker GAP

\section{Freie Demokratische Partei}

\section{s. Zentralverband proletarischer Freidenker}

Gruppe Arbeiterpolitik, 1947 gegr. Zusammenschluß ehem. KPOMitglieder; versuchte in den fünfziger Jahren unter Anknüpfung an die Programmatik Thalheimers* und Brandlers* Perspektiven für die erwartete politische Aktivierung der Arbeiterschaft und die Gründung einer neuen, national eigenständigen kommunistischen Partei zu entwerfen; zum Teil lokaler Einfluß in den Gewerkschaften, 1949/50 maßgebliche Beteiligung an den Protestaktionen der Belegschaft gegen die Demontage der Salzgitter-Werke; nach Übertritten zur SPD I959 aufgelöst, später in einigen Orten neu gegründet. 
GEW

GPU

Gruppe

Neu Beginnen

Gruppe

Internationale

(Spartacus)

$\mathrm{HJ}$

Humanistische Union 196I in München gegr. uberparteiliche Vereinigung zur Verteidigung der freiheidlich-demokratischen Ordnung gegenuber allen Tendenzen zur Errichtung einer weltanschaulich, v. a. konfessionell gebundenen Ordnung.

IAH

IBFG

IG

Internationales revolutionäres sozialistisches Jugendbüro

IRRC

Gewerkschaft Erziehung und Wissenschaft, mit 195270000 Mitgliedern eine der kleineren Gewerkschaften des DGB.

Gosudarstvennoe Politizeskoe Upravlenie, Staatliche Politische Verwaltung, sowjetische Geheimpolizei 1922-1934

gegr. 193 r als geheime Organisation junger oppositioneller Kommunisten und Mitglieder des linken Flügels der SAJ, vgl. Anm. 2 S.Is.

191s gegr. von den radikal-marxistischen Antikriegsgegnern in der SPD (Rosa Luxemburg, Karl Liebknecht, Franz Mehring, Clara Zetkin, August Thalheimer*), spater nach ihrem Organ „SpartacusGruppe benannt; im Mirz 1917 unter Aufrechterhaltung organisawrischer Selbstandigkeit Beitritt zur neugegruindeten USPD, im Dezember 1918 Austritt und - zusammen mit den Bremer Linksradikalen - Grundung der KPD. Der Einfluß der Gruppe Internationale, deren bekannteste Worfuhrer inhafuiert oder an der Front waren, blieb bis Kriegsende gering; ihre Mitgliederzahl wird auf einige hundert bis einige tausend geschătzt.

Hitler-Jugend, 1926 gegr. Jugendorganisation der NSDAP für 14-18jährige, seit 1936 Staatsjugend

Internationale Arbeiterhilfe, 1921 aus Hilfskomitees zur Linderung der Hungersnot in der Sowjetunion entstanden; neben Roter Hilfe und RFB größte Massenorganisation der KPD, widmete sich v.a. der Unterstltzung streikender Arbeiter und wie die Arbeiterwohlfahrt der Sozialfürsorge; 1931 ros 000 Mitglieder, überwiegend parteilose, mit der KPD sympathisierende Arbeiter.

Internationaler Bund Freier Gewerkschaften, im Dezember 1949 gegr. als Gegenorganisation zum kommunistisch beherrschten WGB mit Sitz in Brüssel, umfaßte 195295 Organisationen.

Industriegewerkschaft, erfaßt alle in einem Wirtschaftszweig oder Bereich des Öffentlichen Dienstes beschäftigten Arbeitnehmer unabhängig von ihrer sozialen Stellung und Funktion im Betrieb. Im Unterschied zu den meist auf dem Berufsverbandsprinzip beruhenden Gewerkschaften der Weimarer Republik sind die seit 1949 im DGB zusammengeschlossenen Einzelgewerkschaften nach dem Industrieverbandsprinzip gebildet.

umfaßte die Jugendorganisationen der von 1935 bis 1939 im „Internationalen Büro für revolutionäre sozialistische Einheit “ ${ }^{\text {zusammen- }}$ geschlossenen linkssozialistischen europäischen Parteien: der englischen Independent Labour Party, der ILP Polens, der Sozialistischen Partei Schwedens, der Roten Front Österreichs, der Iberischen Kommunistischen Partei Spaniens.

International Rescue and Relief Committee, später: International Rescue Committee (IRC), $194^{2}$ entstanden aus dem Zusammenschluß einer seit 1933 bestehenden privaten amerikanischen Hilfs- 
ISK

ITF

JLC

Jungbanner

Jungsozialisten

KAPD

KinderfreundeBewegung organisation für Bedrohte der NS-Diktatur und dem 1940 von linkssozialistischen osterreichischen und deutschen Emigranten gegr. „Emergency Rescue Committee“, vgl. S. 17.

Internationaler Sozialistischer Kampfbund, wirkte von $1917 / 18$ bis 1925 unter der Bezeichnung "Internationaler Jugendbund " in der SPD und ihren Nebenorganisationen; nach Unvereinbarkeitsbeschluß durch die SPD 1925/26 Konstituierung als ISK; vgl. Anm. 2 S. 14 sowie die Biographien von Willi Eichler* und Minna Specht*

Internationale Transportarbeiter-Föderation, mit Hilfe der in ihr organisierten Eisenbahner und Seeleute unterstützte die von dem $\mathrm{Hol}$ lănder Edo Fimmen geleitete ITF seit 1933 tatkräftig Widerstandsgruppen in Deutschland und ermöglichte u. a. die Verbreitung illegaler Schriften des ISK, der SAP und der Gruppe Neu Beginnen.

Jewish Labor Committee, 1935 gegr. Zusammenschluß einiger Gewerkschaften der Herren- und Damenkonfektionsbranche, deren meist jüdische Mitglieder aus Osteuropa eingewandert waren; unterstützte die SOPADE und andere sozialistische deutsche Emigrantengruppen in den USA.

\section{Jugendorganisation des Reichsbanners Schwarz-Rot-Gold}

entstanden 1919 aus lokalen Gründungen, 1920 von der SPD als Jugendorganisation für 18-25jährige anerkannt; war 1922-1926 in eine rechte und eine linke Strömung gespalten. Der HofgeismarKreis der rechten Jungsozialisten, der die Organisation bis 1925 beherrschte, verkörperte eine Mischung aus Jugendbewegung, Sozialismus und Nationalbewußtsein; er bejahte nachdrücklich Staat und Nation und versuchte, den parteioffiziellen Marxismus durch einen idealistisch nerneuerten Sozialismus" abzulösen; nach einem deutlichen Linksruck der Jungsozialisten 1925 verließen die Hofgeismarer im Frühjahr 1926 die „Bewegung “ und lösten sich wenig später auf. Die seit 1924 im „Hannoveraner Arbeitskreis “ zusammengeschlossenen marxistisch orientierten Jungsozialisten lehnten sich eng an die SPD-Parteilinken an; seit Ende 1926 erschien mit Unterstützung von Marx Adler, Georg Engelbert Graf und Anna Siemsen die "Jungsozialistische Schriftenreihe ${ }^{\text {". Diese wie Studienaufenthalte an }}$ der von Otto Jenssen und Erich Winkler geleiteten sozialistischen Heimvolkshochschule Tinz bei Gera bildeten die Grundlage einer intensiven Bildungsarbeit; max. 4000 Mitglieder. 1946 neu gegr. als Vertretung der aktiven SPD-Mitglieder im Alter von $18-35$ Jahren.

Kommunistische Arbeiter-Partei (Deutschlands), aus einer Oppositionsströmung der KPD hervorgegangen, im April 1920 gegr. linkskommunistische Partei, in scharfer Gegnerschaft zur Politik der KPD und der Bolschewiki, proklamierte die Verwirklichung des Rätesystems, 192038000 Mitglieder, nach mehrfacher Spaltung seit 1924 Splittergruppe.

sozialdemokratische Erziehungsgemeinschaft, gegr. in Österreich zwischen 1908 und 1917, in Deutschland Anfang der zwanziger Jahre unter maßgeblicher Beteiligung sozialistischer Reformpädagogen; ihre ständigen Helfer organisierten im lokalen Rahmen Spiel- und Arbeitsgruppen für Arbeiterjugendliche im Alter von 
6-14 Jahren, seit 1927 auch überregional sog. Kinderrepubliken, selbstverwaltete Zeltlager mit I-2000 Teilnehmern. Die einheitlich gekleideten Kinderfreunde (blauer Kittel, rotes Halstuch) waren nach Altersgruppen eingeteilt: Nestfalken (6-10 Jahre), Jungfalken (10-12 J.), Rote Falken (12-14J.), anschließend Übergang zur SAJ; Ende 1930 bestanden 788 Kinderfreunde-Ortsgruppen mit 120000 Kindern.

$\mathrm{KJ}, \mathrm{KJD}, \mathrm{KJV}, \mathrm{KJVD}$ Kommunistische Jugend (Deutschlands), 1920 hervorgegangen aus der 1917 gegr. Oppositionellen „Freien Sozialistischen Jugend“ (FSJ), I925 umbenannt in „Kommunistischer Jugendverband Deutschlands" (KJVD), Jugendorganisation der KPD, erfaßte Jugendliche im Alter von I4-23 Jahren, im Herbst 192370000,1929 20000 und 1932 50000 Mitglieder.

KJVO

Kommunistischer Jugend-Verband-Opposition, nach AusschluB der Rechtsopposition aus KPD und KJVD im Dezember 1928 gegr. Jugendorganisation der KPO, zählte 1929 ca 1000 Mitglieder.

$\mathrm{KL}$

KlassenkampfGruppe

Komintern

KP

KPČ

KPD

\section{Konzentrationslager}

Gruppe der marxistischen SPD-Linken um die seit 1927 erscheinende gleichnamige Halbmonatsschrif (Aufl. ca. 1000); die vom Austromarxismus beeinflußte Gruppe trat in der Weimarer Republik für eine konsequente Vertretung der sozialen Interessen der Arbeiterschaft ein; 1928 gegen den Eintritt der SPD in die "Große Koalition", in scharfer Opposition gegen die Wehrpolitik (Panzerkreuzer-Bau) und die Tolerierungspolitik der Partei ab 1930; nach Parteiausschluß ihrer Führer Max Seydewitz ${ }^{*}$ und Kurt Rosenfeld gründete der größte Teil der Linken um den „Klassenkampf" im Oktober 1931 die SAP, eine Minderheit verblieb in der SPD.

Kommunistische Internationale, 1919 gegründeter Zusammenschluß kommunistischer und revolutionärer Parteien mit dem Ziel der Weltrevolution zur Errichtung der Räteherrschaft und der Diktatur des Proletariats, seit dem V.Weltkongreß 1924 weitgehend den Interessen der Sowjetunion unterworfen, 1943 aufgelost.

Kommunistische Partei

Kommunistische Partei der Tschechoslowakei

Kommunistische Partei Deutschlands, gegr. am 30.12. 1918/1. I. I919 als Zusammenschluß von Spartacus-Gruppe und Bremer Linksradikalen; 1919 Ermordung ihrer aus der linken Vorkriegssozialdemokratie kommenden Führer Rosa Luxemburg, Karl Liebknecht, Leo Jogiches; im April 1920 Verlust der Hälfte (5000) der Mitglieder durch Ausschluß der linkskommunistischen Strömung (KAPD); Ende 1920 durch Vereinigung mit den ca. 300000 Mitgliedern der linken USPD Massenpartei, gewann die KPD in der Folgezeit die Mehrzahl der (linken) USPD-Hochburgen im Ruhrgebiet und in Mitteldeutschland; ihre Politik schwankte 1920-1923 zwischen Einheitsfrontbestrebungen mit der SPD und revolutionären Aktionen (1921 Mitteldeutscher Aufstand, 1923 Hamburger Aufstand). In der Krisensituation der Jahre 1922-1924 Radikalisierung der Mitgliedschaft und Ablösung der rechten Führung (Heinrich 
Brandler, August Thalheimer ${ }^{*}$ ) durch die Parteilinke (Ruth Fischer, Arkadij Maslow) im April 1924. Deren Politik (Bolschewisierung der Partei, Kampf gegen die SPD, Gründung kommunistischer Richtungsgewerkschaften) führte zu einem Rückgang des Einflusses der KPD in der Arbeiterschaft (bei der Reichstagswahl vom Dezember 1924 Verlust von x Mill. Stimmen gegenüber dem Frühjahr) und insbesondere in den Gewerkschaften (1922 $88 \mathrm{KPD}$-Delegierte, 1925 3); 1925 zählte die KPD nur noch 125000 Mitglieder. Unter dem Druck der Komintern 1925 Auseinanderbrechen der linken Mehrheit in der KPD in mehrere linke und ultralinke Gruppen (Fischer, Maslow, Korsch u.a.), die bis 1927 aus der Partei ausgeschlossen wurden, und in einen kominterntreuen Flügel um Emst Thälmann. Thälmann, von 1925-1933 KPD-Vorsitzender, stuitzte sich auf die kominterntreue Linke und die Mittelgruppe (Versöhnler) um Ernst Meyer, doch konnten auch die Rechten um Paul Frölich* und Jacob Walcher* wieder Positionen besetzen. Seit 1925 trat die KPD für eine aktive Mitarbeit in den Freien Gewerkschaften ein und betrieb gegenüber der SPD eine gemäßigte Politik, ihr Einfluß blieb in den Jahren der relativen politischen und wirtschaftlichen Stabilität 1925-1928 begrenzt (Reichstagswahl 1928 KPD 10,6\%, SPD 29,8\%). Der Prozeß der Bolschewisierung, der Einschränkung der innerparteilichen Demokratie und der zunehmenden Unterwerfung der KPD unter die Interessen der Sowjetunion setzte sich unter Thălmann fort und führte im Herbst 1928 zur Übernahme der in der Sowjetuntion von Stalin inaugurierten Linkswendung. Auf der Grundlage einer von der Komintern prognostizierten Zuspitzung der Krise des Kapitalismus und bevorstehender Massenkämpfe forcierte die KPD seitdem erneut ihren Kampf gegen die SPD, den wie es nun parteioffiziell hieß - linken Flügel des Faschismus (Sozialfaschismusthese) und gegen die Gewerkschaften (Gründung der RGO). Ca. 6000 Vertreter der rechten Opposition, überwiegend alte Kader der Partei, wurden ausgeschlossen; ein Teil von ihnen gründete im Dezember 1928 die KPO.

In der Weltwirtschaftskrise nahm die KPD seit 1929 einen groBen Aufschwung, vor allem Arbeitslose und jugendliche Arbeiter fühlten sich von ihrer ultralinken Politik angezogen; mit $1932 \mathrm{ca}$. 300000 Mitgliedern und nahezu 6 Mill. Wählern (Reichstagswahl vom November 1932 KPD 16,9\%, SPD 20,4\%) nach NSDAP und SPD drittstärkste Partei.

KPD-Rechte

traten in der KPD für die Einheitsfront mit der SPD, für die Mitarbeit in den Freien Gewerkschaften und für eine aktive Parlamentsarbeit ein, um Teilziele durchzusetzen. Bis 1923 bestimmten sie (Brandler*, Thalheimer*, Walcher* u.a.) die Politik der KPD, 1924-1928 in der Partei nur noch schwach vertreten; 1928/29 überwiegend ausgeschlossen, sammelten sie sich in der KPO.

KPD-Versöhnler

(auch Mittelgruppe) bejahten wie die Rechten eine kommunistische Realpolitik; im Unterschied zu ihnen traten sie jedoch betont für die Führungsrolle der KPdSU in der Komintern ein und versuchten, um jeden Preis in der KPD zu wirken; wichtige Positionen im KPDApparat, bildeten 1926-28 zusammen mit der kominterntreuen Linken unter Ernst Thälmann die KPD-Führung. $1928 / 29$ in Opposi- 
KPD-Linke

KPdSU

KPO

KPÖ

KZ

MdB

MdL

MdR

Mil. Reg.

MK

Neu Beginnen

NKWD

NL

NRW

NS

NSDAP

NY

ÖTV

ÖVP

OKW tion gegen die Linkswendung paßten sie sich nach dem Tod ihres Führers Ernst Meyer im Frühjahr 1930 dem Kurs der Partei zunehmend an.

propagierten vorrangig das kommunistische Endziel, lehnten Übergangsforderungen $a b$ und wollten den gewaltsamen Aufstand vorbereiten; gegen die Einheitsfrontpolitik mit der SPD empfahlen die Linken teilweise die Gewerkschaftsspaltung und betrieben in den Parlamenten Obstruktionspolitik. 1924/25 fuhrten sie unter Ruth Fischer und Arkadij Maslow die Partei. Nach Herausbildung der kominterntreuen Linken 1925 in mehrere Fraktionen gespalten und großtenteils aus der KPD ausgeschlossen, Reste sammelten sich im Fruhjahr 1928 im Leninbund.

Kommunistische Partei der Sowjeaunion

Kommunistische Partei-Opposition, nach der Linkswendung der Komintern und der KPD (Sozialfaschismusthese, Gründung der RGO) im Dezember 1928 von ausgeschlossenen Vertretern der sog. Rechtsopposition um die ehem. KPD-Fuhrer Heinrich Brandler* und August Thalheimer* gegrundet; ca 3500 Mitglieder, uberwiegend in Partei- und Gewerkschaftsarbeit erfahrene, aus der Spartacus-Gruppe und der linken USPD hervorgegangene Funkcionäre; vgl. S. $13-16$.

Kommunistische Partei Österreichs

Konzentrationslager

Mitglied des Bundestages

Mitglied des Landrages

Mitglied des Reichstages

Militärregierung

Mark

s. Gruppe Neu Beginnen

Narodnyj Komissariat Vnutrennich Del, Volkskommissariat für Inneres, sowjetische Geheimpolizei ab 1934.

Nachlaß

Nordrhein-Westfalen

Nationalsozialismus, nationalsozialistisch

Nationalsozialistische Deutsche Arbeiter-Partei, gegr. 1919/20

New York

Gewerkschaft Öffentliche Dienste, Transport und Verkehr, Ende 1952817000 Mitglieder

Österreichische Volkspartei, christlich-konservative Partei

Oberkommando der Wehrmacht 
OMGUS

OSS

P.E.N.-Club

Pfg.

Pgs

Pol.-Leiter

POUM

PV

PW

Reichsbanner Schwarz-Rot-Gold

Revolutionäre Sozialisten Deutschlands
Office of Military Government for Germany, seit Oktober 1945 höchste Behörde für die von den USA besetzten Gebiete Deutschlands.

Office of Strategic Services, im Juni 1942 gegr., vom Militär unabhängiger Geheimdienst mit dem Auftrag, Informationen über die Lage in Deutschland und in den von Deutschland besetzten Gebieten zu sammeln und zu analysieren; in der zentralen Auswertungsstelle in Washington beschäftigte der OSS deutsche Emigranten, die wie Herbert Marcuse oder der Gewerkschaftsjurist Franz L. Neumann der Arbeirerbewegung verbunden waren.

Abk. für engl.: Poets, Playwrights, Editors, Essayists, Novelists, 192 I gegrundete internationale Schriftstellervereinigung; seine Mitglieder verpflichten sich zur Bekämpfung von Rassen-, Klassen- und Völkerhaß und zum aktiven Eintreten für Pressefreiheit und Meinungsvielfalţ, setzt sich für Volkerverständigung und ungehinderten internationalen Gedanken- und Literaturaustausch ein.

Pfennig

Mitglieder der NSDAP: Ende 192527000 , Ende 1928 I 10000, Sept. 1930 300000, Januar 1933 850000, Juni 19332500000 , Juni 1937 $3500000,19436500000$.

Politischer Leiter einer Ortsgruppe oder eines Bezirks der KPD oder der KPO

Partido Obrero de Unificación Marxista, spanische linkssozialistische Partei, 1935 hervorgegangen aus dem Zusammenschluß zweier Parteien, propagierte bei Ausbruch des Spanischen Bürgerkriegs die Bildung einer auf revolutionäre Komitees gestützten Arbeiter- und Bauernregierung, wurde von der KPO und der SAP unterstützt, in ihrer Kritik an der Sowjetunion beeinflußt durch Trotzki; von der Komintern bekämpft als „Agentur des Faschismus ${ }^{\alpha}$ und seit Mai 1937 durch die spanischen Kommunisten unter Mitwirkung der NKWD blutig unterdrückt.

Parteivorstand (der SPD)

Prisoner of War, anglo-amerikanische Bezeichnung für Kriegsgefangener

gegr. 1924 mit dem Ziel, alle auf dem Boden der Republik stehenden Kriegsteilnehmer des Ersten Weltkriegs zum Schutz der Republik und ihrer Einrichtungen zusammenzufassen, 1924 ca. 700000 Mitglieder, davon bekannten sich fast $90 \%$ zur SPD, der Rest verteilte sich auf Mitglieder und Anhänger des Zentrums und der DDP.

gegr. 1935 im Exil von führenden Vertretern der SPD-Parteilinken (Karl Böchel, Siegfried Aufhäuser), die in Opposition zur Politik der SOPADE standen; die RSD forderten den radikalen Bruch mit allen reformistischen Traditionen und hofften, durch die Neugründung einer revolutionären sozialdemokratischen Partei die Einheit der Arbeiterklasse wiederherstellen zu können; 1937 Auflösung und Rückkehr des größten Teils der RSD in die SOPADE.

RFB gegr. Ende Mai 1924 als kommunistische Gegenorganisation zum deutschnationalen Stahlhelm und zum Reichsbanner Schwarz- 
Rot-Gold, I926 ca. I 50000 Mitglieder, darunter viele jugendliche Arbeiter.

RGI

RGO

Rpf.

$\mathrm{RH}$ (D)/Rote Hilfe

RM

Rote Falken

Rote Kämpfer

Roter Frauen- und Mädchenbund

SA

SAJ

$\operatorname{SAP}(D)$
Rote Gewerkschaftsinternationale, gegr. 1919 in Moskau als Zusammenschluß kommunistischer und revolutionärer Gewerkschaften mit dem Ziel der Organisierung der Arbeiter zum Sturz des Kapitalismus.

Revolutionäre Gewerkschafts-Opposition, 1929 gegr. Gewerkschaftsorganisation der KPD, die damit ihre seit 1925 praktizierte Politik der Stärkung des kommunistischen Einflusses in den ADGBGewerkschaften aufgab; mittels der Durchfuhrung von Streiks und deren Ausweitung zu Massenkämpfen sollte die RGO die Revolution vorantreiben, de facto isolierte sie unter den Bedingungen der Weltwirtschaftskrise die kommunistischen Arbeiter und vertiefte die Spaltung der Arbeiterbewegung; 1932 ca. 250000 Mitglieder, darunter ein erheblicher Teil Arbeitslose; am stärksten in Berlin, am Niederrhein und in Sachsen.

Reichspfennig

Rote Hilfe (Deutschlands), I924 gegr., der KPD nahestehende Massenorganisation zur Unterstlitzung politischer Gefangener, 1930 uber 150000 Mitglieder.

Reichsmark

Altersgruppe ( $12-I_{4}$ Jahre) der Kinderfreunde

1930 gegr. Gruppierung um die gleichnamige Zeitschrift; ihre intellektuellen Führer Karl Schröder, Alexander Schwab und Bernhard Reichenbach gingen aus der KAPD hervor; traten als Oppositionsgruppen innerhalb der SPD und seit 1931 auch innerhalb der SAP für eine Aktivierung der Arbeiterschaft und Einheitsfrontpolitik ein; I932 ca. 400 Mitglieder, daruber hinaus zahlreiche Anhänger in der SAJ, bei den Jungsozialisten und Sozialistischen Studenten im Rhein-Ruhr-Gebiet und in Ostsachsen; seit 1932 Umstellung ihrer Gruppen auf konspirative Tätigkeit.

1925 gegr. Frauenorganisation der KPD

Sturmabteilung der NSDAP, 1920 gegr. uniformierte und bewaffnete politische Kampf- und Propagandaorganisation der NSDAP, hatte 193177000 , I933 rd. 700000 vor allem jugendliche Mitglieder, darunter zahlreiche Arbeitslose, wurde von der NSDAP im Straßenkampf und in der Propaganda zur Terrorisierung politischer Gegner eingesetzt.

Sozialistische Arbeiter-Jugend, Jugendorganisation der SPD, 1922 hervorgegangen aus der Verschmelzung von AJ und der „Sozialistischen Proletarierjugend“ der USPD, erfaßte 1922 85000, 1927 55000 und I 93150000 Jugendliche im Alter von I 4- I 8 bzw. ab 1927 20 Jahren; seit 1929 formierte sich in der SAJ eine linke Opposition, ein Teil von ihnen schloß sich I93 I dem SJV an.

Sozialistische Arbeiterpartei (Deutschlands), in Oktober 1931 gegr. von einem Teil der SPD-Linken, hervorgegangen aus der innerpar- 
SBZ

SDS

SDV

SED

SJV (D)

SMA (D)

SOPADE

teilichen Opposition gegen den Panzerkreuzerbau und die Tolerierungspolitik der SPD; 1932 ca. 25000 Mitglieder mit regionalen Schwerpunkten in Schlesien (Breslau), Thüringen, Sachsen (Dresden) und Offenbach, im April 1932 Beitrit der KPO-Minderheit um Jacob Walcher* und Paul Frölich", im Februar 1933 von den Vorstandsmitgliedern Max Seydewitz $z^{*}$ und Kurt Rosenfeld offiziell aufgelöst, kehrten ca. 1500 Mitglieder der Partei den Rücken; die Mehrheit (ca. 15000 ), darunter insbesondere jüngere Mitglieder, arbeitete in kleinen Gruppen illegal weiter; zur Politik der SAP vgl. S. 13-16.

\section{Sowjetische Besatzungszone}

Sozialistischer Deutscher Studentenbund, 1946 gegr. der SPD nahestehender Studentenverband, 196r Unvereinbarkeitsbeschluß durch die SPD.

Sozialistisch-Demokratische Vereinigung Mosbach, 1945 von Funktionären und Mitgliedern der SPD und KPD gegr. Einheitspartei, löste sich 1947 auf; ein kleinerer Teil der Mitglieder schloß sich der AP an.

Sozialistische Einheitspartei Deutschlands, im April 1946 in der SBZ durch Verschmelzung von SPD und KPD entstanden; die SED, die im Herbst 1947 I,8 Mill. Mitglieder zählte, war anfangs eine Massenpartei mit gewissen Einflußmöglichkeiten der Mitgliedschaf, erst 1948/49 erfolgte ihre Veränderung zur marxistisch-leninistischen „Partei neuen Typus“.

Sozialistischer Jugendverband (Deutschlands), Jugendorganisation der SAP, im Oktober 193I von einem Teil der aktivistischen linken SAJ-Opposition gegründet; mit seinen 8-10000 Mitgliedern war der SJV - gemessen an der Mitgliederzahl der SAP - vergleichsweise stark.

\section{Sowjetische Militäradministration (in Deutschland)}

Sozialdemokratische Partei Deutschlands, im Mai 1933 in der CSR von einem Teil des SPD-PV (Otto Wels, Hans Vogel u.a.) gegr. Exilvertretung der SPD, betrachtete sich gegenüber konkurrierenden linkssozialdemokratischen Gruppen als alleinige rechtmäßige Vertretung der Gesamtpartei. Die SOPADE betreute die sozialdemokratischen Emigranten (1936/37 ca. I 500, Anfang 1938 ca. 4000,19398000 ), unterstützte über ihre Grenzsekretariate und Kuriere illegal arbeitende Inlandsgruppen und versuchte mittels ihrer „Deutschland-Berichte" die internationale Öffentlichkeit über die NS-Diktatur aufzuklären; die Bildung einer Einheitsfront mit der KPD lehnte sie ab; Mitte 1938 Übersiedlung nach Paris, später nach London, wo sie 194I eine Kartellverbindung mit der SAP, dem ISK und der Gruppe Neu Beginnen einging; nach Kriegsende unterstützte die SOPADE (Hans Vogel, Erich Ollenhauer, Fritz Heine) Kurt Schumacher.

Sozialistische Jugend Deutschlands Die Falken gegr. 1946, organisatorisch selbständige, der SPD nahestehende Jugendorganisation, umfaßt Kinder und Jugendliche im Alter von 6-2 I Jahren, 1968 rd. I00000 Mitglieder. 
Spartacus-Gruppe

$\mathrm{SP}(\mathrm{D})$

SPÖ

SS

SS-Division

Dirlewanger

Strafbataillon 999

SU

$\mathrm{TH}$ s. Gruppe Internationale (Spartacus)

Sozialdemokratische Partei (Deutschlands), proklamierte in der Revolution $1918 / 19$ wie die sozialdemokratisch geprägten A \& S-Räte die schnelistmogliche Durchführung von Wahlen zu einer verfassunggebenden Nationalversammlung als Voraussetzung fur politische und gesellschaftiche Veränderungen; mit I ,s Mill.Stimmen (37,9\%) stärkste Partei, stellte sie mit Friedrich Ebert den ersten Reichspräsidenten und bildete mit Zentrum und DDP die sog. Weimarer Koalition; aus Entuauschung uber die Politik der SPD in und nach der Revolution Hinwendung groBerer Teile der Arbeiterschaft zur USPD und zur KPD; bei den Reichstagswahlen vom Juni 1920 nur noch 21,6\% (USPD 18,0\%). Seit 1919 Ausbau und Ausdifferenzierung des sozialdemokratischen Organisationsnetzes durch Gründung der AWO, der Kinderfreunde-Bewegung, der Jungsozialisten, des Reichsbanners Schwarz-Rot-Gold, der Arbeitsgemeinschaft sozialdemokratischer Lehrer usw.; im September 1922 Vereinigung mit der USPD, 19201180000 Mitglieder, Anfang 1925 690000, Ende 1931 1009000, regionale Schwerpunkte in Sachsen, Thuringen, Schlesien, Hessen und Hamburg. Programmatische Öffnungsversuche zu einer sozialdemokratischen Volkspartei (Gorlizzer Programm 1921), mit dem Heidelberger Programm 1925 erneute Akzentuierung der traditionell marxistischen Vorkriegsprogrammatik; 1922-1928 mit einer kurzen Ausnahme in Opposition zu bürgerlichkonservativen Reichsregierungen, in diesem Zeitraum Konkretisierung reformorientierter gesellschaftlicher Veränderungsvorstellungen (Kieler Programm 1927). Im Herbst 1928 nach erheblichen Stimmengewinnen in der Reichstagswahl (29,8\%, 9, I Mill. Stimmen) Bildung einer Großen Koalition mit Hermann Müller (SPD) als Reichskanzler; nach Zuspitzung der sozialen Probleme und der Gegensätze innerhalb der Koalition vor dem Hintergrund der Weitwirtschaftskrise im Mărz 1930 Bruch der Großen Koalition, danach Tolerierung der Präsidialregierung Brlining als „kleineres Übel ${ }^{\alpha}$ zur Verhinderung einer nationalsozialistischen Machtubernahme; Versuche parteiinterner Kritiker auf der Linken wie auf der Rechten, die SPD zu einer aktiveren Oppositionspolitik zu bewegen, konnten sich 1932 nicht durchsetzen. Trotz der sozialen Krisenfolgen und politischen Radikalisierung blieb die Wählerschaft der SPD als Partei überwiegend der gelernten Facharbeiter relativ stabil: 1930 8,s Mill., Nov. 1932 7,2 Mill., März 1933 7,2 Mill.

\section{Sozialistische Partei Österreichs}

Schutzstaffel der NSDAP, 1925 gegr. Sonderorganisation zum Schutz Hitlers und anderer NSDAP-Funktionäre, wurde nach 1933 zum wichtigsten politischen Sicherheits- und Terrorinstrument des NS-Regimes.

Vgl. Anm. 27, S. 26.

Vgl. Anm. 27, S. 26.

Sowjetunion

Technische Hochschule 
Touristenverein Die Naturfreunde

UAP (D)

UdSSR

Ultralinks

UN

Union deutscher sozialistischer Organisationen in Großbritannien

UNESCO

USA

USP (D) gegr. 1895 in Wien, nahm nach dem Ersten Weltkrieg auch in Deutschland einen raschen Aufschwung. Das Vereinsleben war durch Familien- und Gemeinschaftswandern, gesellige Veranstaltungen und politisch-wissenschaftliche Vorträge gekennzeichnet. Der besondere Stolz der Naturfreunde galt den von ihnen meist in Zusammenarbeit mit anderen lokalen Arbeiterorganisationen in Eigeninitiative gebauten Hütten und Heimen, von denen es 1929 $241 \mathrm{gab}$. In der Reichsleitung und in der Gesambewegung dominierten Sozialdemokraten.

Unabhängige Arbeiterpartei (Deutschlands), im März I95 I gegr. von einer Gruppe ausgeschlossener KPD-Mitglieder, Trotzkisten und einigen „heimatlosen Linken“, national „unabhängige“ kommunistische Partei. Trotz erheblicher finanzieller Zuwendungen seitens Jugoslawiens kam die UAP nicht über 400 Mitglieder hinaus, 1952 löste sie sich auf.

Union der Sozialistischen Sowjetrepubliken

Bezeichnung für die Politik der KPD $1924 / 25$ und 1928/29-1935. United Nations

am 19.3. 194I gegr. Zusammenschluß aus SOPADE, SAP, ISK, Gruppe Neu Beginnen und der "Landesgruppe deutscher Gewerkschafter in Großbritannien “. Die Zusammenarbeit in der von Hans Vogel (SPD), Erwin Schoettle (Neu Beginnen), Willi Eichler* (ISK) und Hans Gottfurcht (Gewerkschaften) geleiteten „Union“ ließ in den folgenden Jahren die organisatorischen Abgrenzungen und politischen Differenzen in den Hintergrund treten und bildete eine Etappe auf dem Weg zur Reintegration der sozialistischen Gruppen und Kleinparteien in die SPD nach 1945.

United Nations Educational, Scientific and Cultural Organization, I 945 gegr. Sonderorganisation der UN zur Förderung der internationalen Zusammenarbeit auf den Gebieten der Erziehung, Wissenschaft und Information.

United States of America

Unabhängige Sozialdemokratische Partei (Deutschlands), nach Ausschluß der Opposition aus der SPD gegr. am 6./7.4. 1917 als $\mathrm{Zu}$ sammenschluß der sozialdemokratischen Kriegsgegner, wandte sich vehement gegen die Bewilligung der Kriegskredite und die Burgfriedenspolitik der SPD; politisch heterogen, umfaßte sowohl Anhänger des alten Parteizentrums (Karl Kautsky), Revisionisten (Eduard Bernstein, Kurt Eisner), gemäßigte radikale Linke (Hugo Haase, Rudolf Hilferding) und - organisatorisch selbständig (bis 1918) die radikale Linke der „Gruppe Internationale " (Spartacus); im November/Dezember 1919 Beteiligung am Rat der Volksbeauftragten, erhielt bei der Wahl zur Nationalversammlung im Januar $19197,6 \%$ (SPD 37,9\%) der Stimmen, im März 1919300000 Mitglieder (SPD ca. I Mill.). I919/20 Sammelbecken der von der Politik der SPD enttäuschten und sich radikalisierenden Massen; Reichstagswahl vom Juni 1920 USPD $18,0 \%$, SPD $21,6 \%$. Auf dem Höhepunkt ihres Einflusses (Herbst 1920900000 Mitglieder) beschloß ein außerordentlicher USPD-Parteitag im Oktober 1920 mit ZweidrittelMehrheit die Annahme der 21 Bedingungen der Komintern und die 
VEB

Versöhnler

VVN

WAV

WC

WDR

WGB

Z

ZdA

Zentralverband proletarischer Freidenker
Vereinigung mit der KPD. Jeweils ca. ein Drittel der Mitglieder trat zur KPD über, organisierte sich nicht mehr parteipolitisch bzw. verblieb in der USPD, die im September 1922 in die SPD zurückkehrte.

Volkseigener Betrieb in der DDR

s. KPD-Versöhnler

Vereinigung der Verfolgten des Naziregimes, 1947 gegr. Zusammenschluß ehem. Widerstandskämpfer, infolge seiner Anlehnung an die Politik der KPD/SED 1948 Austritt nahezu aller Sozialdemokraten; seit den sechziger Jahren arbeiten in der VVN, die sich mit Ausstellungen und Publikationen uber NS-Verbrechen und den antifaschistischen Widerstand an die Öffentlichkeit wendet, in größserem Maße Parteilose und Mitglieder anderer Parteien mit.

Wirtschaftliche Aufbau-Vereinigung, 1945 in Bayern begr., eine stark mittelständisch geprägte Führerpartei mit populistischen Zïgen.

Workmen's Circle, Abkürzung für Workmen's Sick and Death Benefit Fund of the United States of America, Inc.; vgl. S. $16 \mathrm{f}$.

Westdeutscher Rundfunk

Weltgewerkschaftsbund, 1945 in London gegrindete internationale Gewerkschaftsorganisation, seit 1949 unter kommunistischem Einfluß.

Deutsche Zentrumspartei, 1945 neben der CDU von ehemaligen Mitgliedern des katholischen Zentrums der Weimarer Republik gegr., betont konfessionell, föderalistisch, mittelstăndisch, sozialreformerisch im Sinne der christlichen Soziallehre, erreichte bei Wahlen in Nordrhein-Westfalen 1947 knapp ro Prozent der Stimmen; 1946 110000, 195035000 Mitglieder.

Zentralverband der Angestellten, vor 1919: Zentralverband der Handlungsgehilfen, seit 1917 der ${ }_{n}$ Arbeitsgemeinschaft freier Angestelltenverbände" angeschlossen; umfaßte Angestellte aus Verwaltung und kaufmännischen Berufen, unter ihnen viele Frauen; 1920 400000 Mitglieder, 1925 is 3000.

gegr. 1908 in Eisenach mit der Zielsetzung, den politischen und ökonomischen Kampf der SPD durch geistige und antikirchliche Aufklärung zu unterstützen; verpflichtete seine Mitglieder zu politischer und gewerkschaftlicher Organisation. 1922 Verschmelzung mit kleineren Verbänden zur "Gemeinschaft proletarischer Freidenker" $(\mathrm{GpF})$, deren Schwerpunkte in Sachsen, Thüringen und in Rheinland-Westfalen lagen; 1924 100000 Mitglieder, zu einem erheblichen Teil Kommunisten. 1926/27 Verschmelzung der GpF mit dem sozialdemokratischen "Verein der Freidenker für Feuerbestattung ${ }^{\text {" }}$ zum „Verband für Freidenkertum und Feuerbestattung e.V.“. Ein Jahr später hatte die proletarische Freidenkerbewegung, deren Aktivitäten neben ihrer Funktion als Sterbekasse v.2. auf schulpolitischem Gebiet lagen, über 500000 Mitglieder, davon waren ca. 45000 in der KPD organisiert

Zentralkomitee, höchstes Führungsgremium der KPD. 